\title{
Registro de la presencia de mamíferos medianos en dos zonas del municipio de Cuetzalán, en la Sierra Norte de Puebla
}

\author{
Lorena Silverio Polo ${ }^{1}$ y O. Eric Ramírez-Bravo*2,3,4
}

Introduction: Northern Puebla has a high mammal diversity due the large variety of ecosystems present. However, there is no information about their distribution or their response to fragmentation. Thus, we registered medium sized mammals in two areas with different degree of disturbance. Rancho el Paraiso which focus on cattle ranching and the Tecomateno Private Reserve which conserves habitat in the municipality of Cuetzalan, Puebla.

Methods: From September 2012 to February 2013 we used transects to find tracks and scats and camera traps baited with a mix of sardine and lard. Additionally we undertook semi-structured interviews to hunters and exhunters with the aid of cards with images of the species potentially distributed in the area.

Results: We carried out 12 field visits in each site, accumulating a total of 163 camera nights and walking 27 km in Rancho el Paraiso, while in the Tecomateno Reserve, we accumulated 145 camera nights and walked 22.5 $\mathrm{km}$. Our field efforts resulted in the detection of six species for the Tecomateno Reserve and four for Rancho el Paraiso. Our semi-structured interviews documented the presence of 13 species for the Tecomateno Reserve and 11 for Rancho el Paraíso.

Discussion and Conclusions: This study provides information on the mammals of Cuetzalan and their response to different levels of disturbance. It was expected to find a higher diversity in Rancho el Paraiso because it is located away from communities, however, it is used more intensively. We consider that interviews are a reliable cost effective tool to undertake rapid biodiversity assessments. These results also suggest that private nature reserves may be a good option to conserve biodiversity in fragmented areas.

Key words: disturbance, mesopredators, otter, sampling, tropical montane cloud forest

\section{Resumen}

El norte de Puebla cuenta con una gran diversidad de mamíferos debido a la gran variedad de ecosistemas que presenta. Sin embargo, no se cuenta con información sobre su distribución ni sobre su respuesta a la fragmentación. Por lo anterior, se registró la presencia de mamíferos medianos en dos zonas con diferente grado de perturbación. Rancho el Paraíso de vocación ganadera y en Reserva Privada Tecomateno que está destinada a la conservación de hábitat en el municipio de Cuetzalán, Puebla.

\footnotetext{
'Instituto Tecnológico Superior de Zacapoaxtla Puebla, Carretera Acuaco - Zacapoaxtla Km. 8, Col. Totoltepec, C.P. 73680, Zacapoaxtla Puebla. E-mail: (LSP).

${ }^{2}$ Durrell Institute for Conservation Ecology, Marlowe Building, University of Kent, Canterbury, Kent, CT2 7NR, England. E-mail: ermex02@yahoo.com (OERB).

${ }_{3}^{3}$ Departamento de Ciencias Químico-Biológicas, Universidad de las Américas, Puebla, Santa Catarina Mártir, Sin Número, Cholula, Puebla. CP. 72820, México. E-mail: ermex02@yahoo.com (OERB).

${ }^{4}$ CREANATURA A.C., Calle Quetzalcoatl No. 5, 2da. Sección de Quetzalcóatl, Puebla, Puebla. C.P.72960. E-mail: ermex02@ yahoo.com (OERB).

*Corresponding author
} 
De septiembre 2012 a febrero 2013 su utilizaron transectos para la búsqueda de huellas y excretas y cámaras trampa cebadas con una mezcla de sardina y manteca de cerdo. Adicionalmente, se elaboraron entrevistas semi-estructuradas a cazadores y ex-cazadores con ayuda de tarjetas con imágenes de las especies de distribución potencial.

Se realizaron en total 12 salidas por sitio, acumulando un total de 163 noches trampa y $27 \mathrm{~km}$ recorridos en el Rancho el Paraíso y 145 noches trampa y $22.5 \mathrm{~km}$ recorridos en la Reserva Tecomateno. Durante el trabajo de campo se detectaron seis especies para la Reserva Tecomateno y cuatro para el Rancho El Paraíso. Mediante las entrevistas, se documentó la presencia de 13 especies para la Reserva Tecomateno y de 11 en el Rancho El Paraíso.

Este estudio provee información sobre la mastofauna de Cuetzalán y su respuesta ante los distintos grados de perturbación. Se esperaba encontrar una diversidad mayor en el Rancho el Paraíso ya que se encuentra más alejado de los núcleos de población sin embargo; presenta una mayor intensidad de uso. Se considera que las encuestas son una buena herramienta para determinar la biodiversidad de un sitio a bajo costo y de manera rápida. Estos resultados nos permiten considerar que las reservas naturales privadas pueden ser una buena opción para el mantenimiento de la biodiversidad en zonas fragmentadas.

Palabras clave: bosque mesófilo de montaña, mesodepredadores, muestreo, nutria, perturbación

Introducción

La Sierra Madre Oriental presenta una gran diversidad de flora y fauna (Luna et al. 2004) y cuenta con ecosistemas como el bosque mesófilo de montaña que se consideran de los ecosistemas con mayor diversidad promedio de mamíferos en México (Fa y Morales 1993). Uno de los lugares menos estudiados a lo largo de esta cordillera es la Sierra Norte de Puebla, que a pesar de ser una zona que presenta una gran diversidad biológica (Ferrusquia 1993) no cuenta con estudios que describan la riqueza de mamíferos (Ramírez-Bravo et al. en proceso). Menos aún están documentadas las respuestas de los mamíferos ante las perturbaciones que se presentan en esta región pero, se tiene la hipótesis de que la diversidad será mayor en zonas alejadas a los núcleos de población. Por lo anterior, se registró la presencia de mamíferos medianos en dos zonas con diferente grado de perturbación, Rancho "el Paraíso" destinado a la cría de ganado pero alejado de los núcleos de población y la reserva natural privada "Tecomateno" destinada a la conservación de hábitat pero cercana a los núcleos urbanos dentro del municipio de Cuetzalán, Puebla.

Zona de Estudio. El Municipio de Cuetzalán del Progreso se localiza en la Sierra Norte de Puebla entre los paralelos $-19^{\circ} 57^{\prime} 00^{\prime \prime}$ y $20^{\circ} 05^{\prime} 18^{\prime \prime} \mathrm{N}$ y los meridianos $97^{\circ} 24^{\prime}$ 36" y -970 34' 5" W. Su altitud va desde los 320 hasta los 1,500 m (Carrillo-Ruíz y Morón 2003). Presenta dos tipos de climas, semi-cálido húmedo con lluvias todo el año y cálido húmedo con lluvias todo el año con un rango de temperatura de $18{ }^{\circ} \mathrm{C}$ a $26{ }^{\circ} \mathrm{C}$ (INEGI 2009). Presenta asociaciones de pino-encino, bosque tropical sub caducifolio, y bosque mesófilo de montaña (Rzedowski, 1978); sin embargo, el municipio ha 
perdido la mayor parte de sus zonas boscosas (64 \%; INEGI 2009; Figura 1). El trabajo de campo se llevó a cabo en dos zonas con diferente grado de perturbación: Rancho “EI Paraíso" con una superficie de 50 ha de las cuales aproximadamente el $80 \%$ se dedica a actividades ganaderas mientras que la reserva natural privada "Tecomateno" cuenta con 30 ha y está dedicada a la conservación del hábitat (Figura 2).

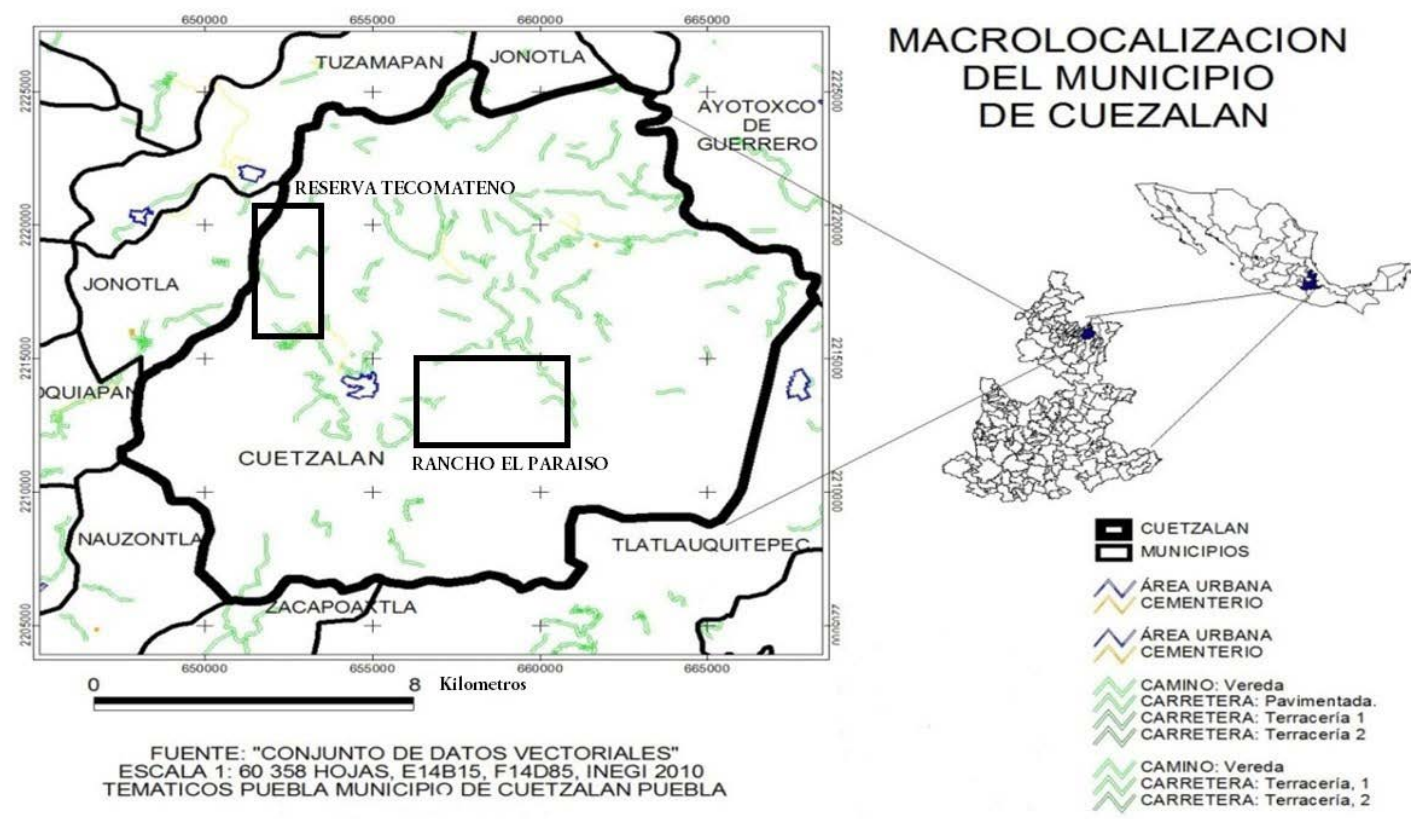

Figura 1. Municipio de Cuetzalán y ubicación de las zonas de estudio.

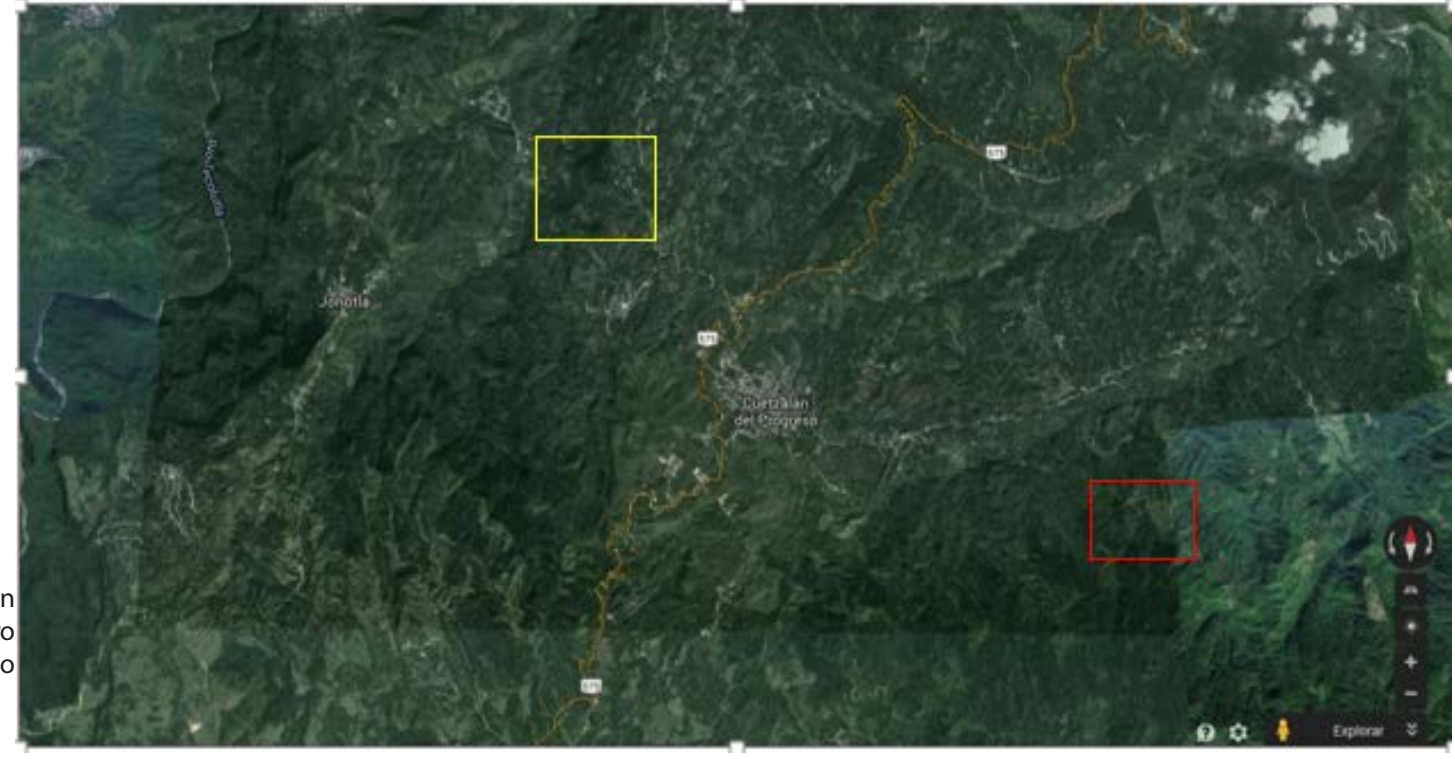

El trabajo de campo se llevó a cabo durante el periodo de septiembre 2012 a febrero 2013 utilizando transectos para la búsqueda de huellas y excretas. Estos fueron trazados al azar con la finalidad de cubrir aproximadamente el $10 \%$ de cada uno (Sanchez-Lalinde y Perez-Torres 2008). Cabe destacar que en el caso del Rancho el Paraíso, el muestreo cubrió de manera similar los potreros y parches de bosque. Adicionalmente, se colocaron nueve estaciones de foto trampeo con una distancia mínima de $1 \mathrm{~m}$ y cada trampa se cebó con una mezcla de sardina y manteca de cerdo. Las cámaras (Bushnell, Trophy Cam) 
fueron revisadas cada quince días, para confirmar su buen funcionamiento y para cambiar memorias de almacenamiento o pilas cuando era necesario. Adicionalmente, se elaboraron entrevistas semi-estructuradas a 13 cazadores y ex-cazadores en cada zona, utilizando el método de entrevistas de Martin (1985) utilizando tarjetas con imágenes de las especies de distribución potencial.

\section{Resultados}

Se realizaron en total 12 salidas por sitio, acumulando un total de 163 noches trampa y $27 \mathrm{~km}$ recorridos (nueve transectos de $3 \mathrm{~km}$ ) en el Rancho el Paraíso y 145 noches trampa y $22.5 \mathrm{~km}$ recorridos (nueve transectos de $2.5 \mathrm{~km}$ ) en la Reserva Tecomateno. En total se encontraron seis especies para la Reserva Tecomateno y cuatro para el Rancho el Paraíso. La zorra gris (Urocyon cinereoargenteus), es la especie mejor representada siendo la que presenta un éxito de captura mayor. Mediante las entrevistas, se documentó la presencia de 13 especies para la Reserva Tecomateno y de 11 en el Rancho El Paraíso estando ausentes la nutria (Lontra longicaudis) y el jaguarundi (Herpailurus yagouaroundi) que se consideran especies amenazadas (Tabla 1).

\begin{tabular}{|c|c|c|c|c|c|c|}
\hline \multirow[b]{2}{*}{ Especie } & \multicolumn{3}{|c|}{ Rancho el Paraíso } & \multicolumn{3}{|c|}{ Reserva natural privada Tecomateno } \\
\hline & Cámara trampa & Rastros & Entrevistas & Cámaras trampa & Rastros & Entrevistas \\
\hline Armadillo (Dasypus novemcinctus) & $\mathrm{x}$ & & $\mathrm{x}$ & $x$ & & $x$ \\
\hline Coatí (Nasua narica) & $x$ & & $x$ & $x$ & & $x$ \\
\hline Jaguarundi (Herpailurus yagouaroundi) & & & & & & $x$ \\
\hline Mapache (Procyon lotor) & & $x$ & $x$ & & $x$ & $x$ \\
\hline Martucha (Potos flavus) & & & $x$ & & & $x$ \\
\hline Nutria (Lontra longicaudis) & & & & & & $x$ \\
\hline Ocelote (Leopardus pardalis) & & & $x$ & $x$ & & $x$ \\
\hline Oso hormiguero (Tamandua mexicana) & & & $x$ & & & $x$ \\
\hline Puerco espín (Coendou mexicanus) & & & $x$ & & & $x$ \\
\hline Tlacuache (Didelphis virginiana) & & & $x$ & $x$ & & $x$ \\
\hline Tigrillo (Leopardus wiedii) & & & $\mathrm{x}$ & & & $x$ \\
\hline Zorra gris (Urocyon cinereoargenteus) & $\mathrm{x}$ & $\mathrm{x}$ & $\mathrm{x}$ & $x$ & $\mathrm{x}$ & $x$ \\
\hline Zorrillo (Mephitidae) & & & $x$ & & & $x$ \\
\hline Total & 3 & 2 & 11 & 5 & 2 & 13 \\
\hline
\end{tabular}

Tabla 1. Exito de captura para cada especie encontrada en cada sitio.

\section{Discusión}

La Sierra Norte de Puebla se caracteriza por tener poca información mastofaunística por lo que este tipo de estudios es de suma importancia para poder llevar a cabo un manejo adecuado en el área. La diversidad encontrada con los transectos y las cámaras trampa se puede considerar baja en comparación con otros estudios que han encontrado una diversidad de 10 especies utilizando los mismos métodos (Martínez 2011). Esta diferencia puede deberse a que no se hizo el muestreo para mamíferos arbóreos como oso hormiguero y puerco espín que fueron reportados en las entrevistas y se ha confirmado su presencia en zonas aledañas (Ramírez-Bravo 2012). La mención de la nutria responde a que la Reserva Natural Tecomateno se encuentra junto a un río donde se ha reportado la existencia de la especie (Ramírez-Bravo et al. 2013). 
Se esperaba encontrar una diversidad mayor en el Rancho el Paraíso ya que se encuentra más alejado de los núcleos de población. Sin embargo, el uso de la tierra en ambos sitios varía ya que aproximadamente el $80 \%$ de este último está destinado a la producción agrícola y el resto se del terreno se encuentra en forma de parches de bosque mesófilo mientras que la Reserva Natural Tecomateno es una zona que destina cerca del 80 $\%$ de su superficie a la conservación del hábitat. Esto ha sido documentado previamente en estudios que han relacionado la riqueza de especies con la intensidad de las actividades antropogénicas y no con la proximidad a núcleos urbanos (Ordeñana et al. 2010).

Se puede apreciar que en ninguno de los sitios se obtuvieron registros de depredadores tope que puede deberse al cambio de uso de suelo para el cultivo de café (Evangelista et al. 2010) ya que las actividades antropogénicas son la principal causa de su desaparición (Woodroffe y Ginsberg 1998). Este hecho puede ocasionar la liberación de los mesodepredadores que en muchos casos se ven beneficiados al incrementar el número de fuentes de alimento (Beasley et al. 2007; Urquiza-Haas et al. 2009). En las dos zonas de estudio se evidencia este fenómeno ya que la zorra gris, al ser una especie generalista tiene un alto índice de detección como en otras zonas (Lorenzana-Piña et al. 2004).

Conclusión. Este estudio provee más información sobre la mastofauna de Cuetzalán y su respuesta ante los distintos grados de perturbación. A pesar de que en el trabajo de campo no se obtuvieron las mismas especies que en las encuestas, se puede decir que estas son una buena herramienta para determinar la biodiversidad de un sitio a bajo costo. Los resultados demuestran que las zonas con alto grado de perturbación tienen una menor diversidad de mastofauna en comparación de los conservados. Sin embargo, llama la atención que en el Rancho el Paraíso que se encuentra altamente perturbado exista una gran diversidad de mamíferos medianos que puede responder a que cuenta con parches de bosque mesófilo y está alejado de los núcleos urbanos. Finalmente, se considera que las reservas naturales privadas pueden ser una buena opción para el mantenimiento de la biodiversidad en zonas fragmentadas.

\section{Literatura citada}

Beasley, J. C., T. L. Devault, y O. E. Rhodes JR. 2007. Home-range attributed of raccoons in a fragmented agricultural region of northern Indiana. Journal of Wildlife Management 71:844-850.

Carrillo Ruiz, H., y M. A. Morón. 2003. Fauna de Coleóptera Scarabaeoidea de Cuetzalán del Progreso, Puebla, México. Acta Zoológica Mexicana nueva serie 88:87-121.

Evangelista, V., J. López, J. Caballero, y M. A. Martínez. 2010. Patrones espaciales de cambio de cobertura y uso del suelo en el área cafetalera de la sierra norte de Puebla. Boletín del instituto de geografía. Universidad Nacional Autónoma de México 72:23-38.

Fa, J. E. y L. M. Morales. 1993. Patterns of mammalian diversity in Mexico. Pp. 319-361 en Biological Diversity of Mexico: Origins and distribution (Ramamoorthy, T. P., R. Bye, A. Lot, y J. Fa. eds.). Oxford University Press, New York, EE.UU.

Ferrusquía, I. 1993. Geology of México: a synopsis. Pp. 3-107 en Biological diversity of Mexico: Origins and distribution (Ramamoorthy, T. P., R. Bye, A. Lot, y J. Fa. eds.). Oxford University Press, New York, EE.UU.

INEGI. 2009. Prontuario estadístico del municipio de Cuetzalán del Progreso. 
Lorenzana Piña, G., R. E. Castillo Gámez, y C. López Gonzales. 2004. Distribution, habitat, association and activity patterns of medium and large sized mammals of Sonora, Mexico. Natural Areas Journal 24:354-357.

Luna, I., J. Morrone, Y D. Espinosa. 2004. Biodiversidad de la Sierra Madre Oriental. Universidad Nacional Atónoma de México -Comisión Nacional para el Uso y Conocimiento de la Biodiversidad. Distrito Federal, México.

Martin, G. J. 1985. Ethnobotany: a methods manual, Chapman y Hall. New York, EE.UU.

Martínez, M. 2011. Inventario de mastofauna mediana y grande, empleando métodos indirectos en campamento Reserva Azul, el Cuichat, Cuetzalán del Progreso Puebla. Memoria de Residencias de Licenciatura en Biología. Instituto Tecnológico Superior de Zacapoaxtla.

Ordeñana, M. A., K. R. Crooks, E. E. Boydston, R. N. Fisher, L. M. Lyren, S. Siudyla, C. D. HaAs, S. Harris, S. A. Hathaway, G. M. Turschak, A. K. Miles, y D. H. Van Vuren. 2010. Effects of urbanization on carnivore species distribution and richness. Journal of Mammalogy 91:1322-1331.

Ramírez-Bravo, O.E. 2012. New records of the Mexican hairy porcupine (Coendou mexicanus) and tamandúa (Tamandua mexicana) in Puebla, Central Mexico. Western North American Naturalist 72:93-95.

Ramírez-Bravo, O. E., P. L. Moreno Barrera, y L. Hernández-Santín. 2013. Public participation as an aid to conserve little known species: The case of the neotropical otter (Lontra longicaudis, Olfers, 1818) in Central Mexico. IUCN Otter Specialist Group Bulletin 30:37-41,

Rzedowski, J. 1978. Vegetación de México. Limusa. Distrito Federal, México.

Sánchez-Lalinde, C., y J. Pérez-Torres. 2008. Uso de Hábitat de carnívoros simpátricos en una zona de bosque seco tropical de Colombia. Mastozoología Neotropical 15:67-74

Urquiza-HaAs, T., C. A. Peres, y P. M. Dolman. 2009. Regional scale effects of human density and forest disturbance on large-bodied vertebrates throughout the Yucatán Peninsula, Mexico. Biological Conservation 142:134-148.

Woodroffe, R., y J. R. Ginsberg. 1998. Edge effects and the extinction of populations inside protected areas. Science 280:2126-2128.

Sometido: 24 de septiembre de 2013

Revisado: 10 de diciembre de 2013

Aceptado: 12 de diciembre de 2014

Editor asociado: Jesús Maldonado 